Ann. Inst. Statist. Math.

Vol. 47, No. 4, 601-619 (1995)

\title{
AREA-INTERACTION POINT PROCESSES
}

\author{
A. J. BADDELEY ${ }^{1}$ AND M. N. M. VAN LIESHOUT ${ }^{2}$ \\ ${ }^{1}$ Department of Mathematics, University of Western Australia, \\ Nedlands WA 6009, Australia and \\ Department of Mathematics and Computer Science, University of Leiden, The Netherlands \\ 2 Department of Statistics, University of Warwick, Coventry CV4 7AL, U.K.
}

(Received December 6, 1993; revised January 30, 1995)

\begin{abstract}
We introduce a new Markov point process that exhibits a range of clustered, random, and ordered patterns according to the value of a scalar parameter. In contrast to pairwise interaction processes, this model has interaction terms of all orders. The likelihood is closely related to the empty space function $F$, paralleling the relation between the Strauss process and Ripley's $K$-function. We show that, in complete analogy with pairwise interaction processes, the pseudolikelihood equations for this model are a special case of the Takacs-Fiksel method, and our model is the limit of a sequence of auto-logistic lattice processes.
\end{abstract}

Key words and phrases: Clustering, empty space statistic, Hough transform, inhibition, $K$-function, lattice process limits, Markov point processes, nearest neighbour distance distribution, pairwise interaction, penetrable sphere model, pseudolikelihood, spatial statistics, spherical contact distance distribution, stationary point process, Strauss model, Takacs-Fiksel method.

\section{Introduction}

Since the introduction of Markov point processes in spatial statistics (Kelly and Ripley (1976), Ripley and Kelly (1977)) (the very similar concept of a Gibbs point process was already known in statistical physics (Ruelle (1969), Chapter 3, Preston (1976))) attention has focused on the special case of pairwise interaction models. These provide "a large variety of complex patterns starting from simple potential functions which are easily interpretable as attractive and/or repulsive forces acting among points" (Mase (1990)). A great deal is understood about pairwise interaction models because they are very natural with respect to the derivation of conditional probabilities, Papangelou conditional intensities and Palm distributions; they are simple exponential families whose sufficient statistics are often related to the popular $K$-function; and they are very amenable to simulation and iterative statistical methods.

However, pairwise interaction processes do not seem to be able to produce clustered patterns in sufficient variety. The original clustering model of Strauss 
(1975) turned out (Kelly and Ripley (1976)) to be non-integrable for parameter values $\gamma>1$ corresponding to the desired clustering; Gates and Westcott (1986) showed that partly-attractive potentials may violate a stability condition, implying that they produce extremely clustered patterns with high probability; and recent simulation experiments by Møller (1994) suggest that the behaviour of the Strauss model with fixed $n$ undergoes an abrupt transition from "Poisson-like" patterns to tightly clustered patterns rather than exhibiting intermediate, moderately clustered patterns.

In this paper we introduce a family of Markov point processes that yield both moderately clustered and moderately ordered patterns. They can be described as having interactions of infinite order. In the simplest case the probability density of a point pattern $\boldsymbol{x}=\left\{x_{1}, \ldots, x_{n}\right\}(n \geq 0)$ in a window $A \subseteq \mathbb{R}^{2}$ is defined to be

$$
p(\boldsymbol{x})=\alpha \beta^{n} \gamma^{-u(x)}
$$

where $u(\boldsymbol{x})$ is the area of the plane set formed by taking the union of discs of radius $r$ centred at the points $x_{i}$. Here $\beta, \gamma, r>0$ are parameters and $\alpha$ is the normalising constant. Compare this with the pairwise-interaction Strauss process in the same situation,

$$
p(x)=\alpha \beta^{n} \gamma^{s(x)}
$$

where $s(x)$ is the number of pairs of distinct points $x_{i}, x_{j}$ that lie within a distance $r$ of one another. Both densities reduce to a Poisson process when $\gamma=1$, and exhibit ordered patterns for $0<\gamma<1$. Our process (1.1) is well-defined for all values of $\gamma>0$ and produces clustering when $\gamma>1$. The clustered case $\gamma>1$ of (1.1) is identical to the 'penetrable sphere model' of liquid-vapour equilibrium proposed by Widom and Rowlinson (1970), see also Hammersley et al. (1975) or Rowlinson $(1980,1990)$. Our Definition 1 embraces both clustered and ordered types and Definition 2 below is a further generalisation to non-spherical shapes and non-uniform measures. Figure 1 shows simulated realisations of (1.1).

It is useful to note that computation of $u(\boldsymbol{x})$ is easy in an image processing context, using the distance transform algorithm (Rosenfeld and Pfalz (1968)).

The plan of the paper is as follows. In Section 2 we define the process and check that it is integrable for all parameter values. We show that it is a Markov point process with interactions of infinite order, and give various physical interpretations. In Section 3 we prove that the process satisfies a stability condition and that there is a corresponding stationary Gibbs process on $\mathbb{R}^{d}$. Section 4 briefly discusses simulation techniques.

In Section 5 we consider statistical inference. First we show that (1.1) is connected to the popular empty space statistic $F$ in the same way that the Strauss process (1.2) is related to Ripley's $K$-function. We show that pseudolikelihood inference for the area-interaction process is a special case of the Takacs-Fiksel method, analogous to the situation for pairwise interaction processes (Diggle et al. (1994), Särkkä (1989), Ripley (1989)). Finally in Section 6 we prove that the area-interaction process is the limit (weakly and in total variation) of a sequence of autologistic lattice processes, extending the limit theorem of Besag et al. (1982). 

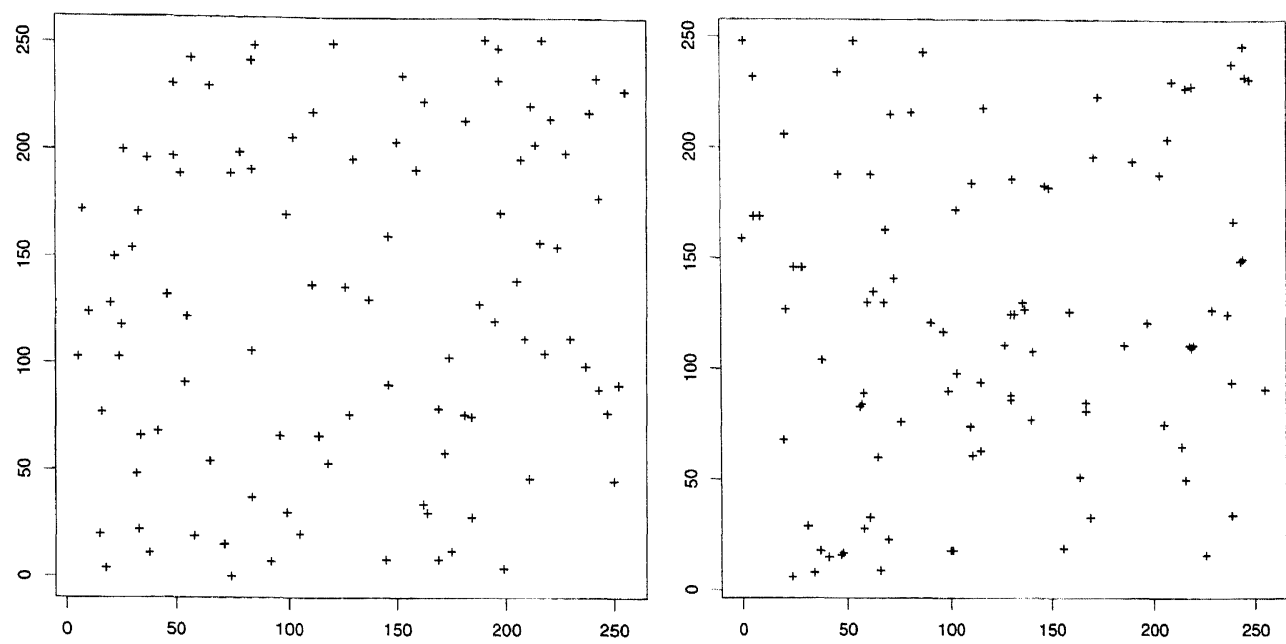

Fig. 1. Simulated realisations of an area-interaction process conditional on $n=100$ points, with $r=5$ in a window of size $256 \times 256$. Left: ordered pattern, $\gamma=0.9711$, $\gamma^{-25 \pi}=10 ;$ Right: clustered pattern, $\gamma=1.02975, \gamma^{-25 \pi}=0.1$.

\section{Definition of process}

\subsection{Preliminaries}

As usual for Gibbs point processes we treat separately the cases of a finite point process (say, points in a bounded region $A \subseteq \mathbb{R}^{d}$ ) and a stationary point process on $\mathbb{R}^{d}$.

The formal construction of finite Gibbs point processes is described in Daley and Vere-Jones ((1988), p. $121 \mathrm{ff})$, Preston (1976) or e.g. Section 2 in Baddeley and Møller (1989). Briefly, let $\mathcal{X}$ be a locally compact complete separable metric space (typically $\mathbb{R}^{d}$ or a compact subset). A realisation of a finite point process is a finite set of points

$$
x=\left\{x_{1}, \ldots, x_{n}\right\}, \quad x_{i} \in \mathcal{X}, \quad n \geq 0 .
$$

The space of all possible realisations shall be identified with the space $\Re^{f}$ of all integer-valued measures on $\mathcal{X}$ which have finite total mass and are simple (do not have atoms of mass exceeding 1 ). Write $n(\boldsymbol{x})$ for the total mass (=total number of points), and $x_{B}$ for $x$ restricted to $B \subseteq \mathcal{X}$. The $\sigma$-algebra $\mathcal{N}^{f}$ on $\Re^{f}$ is the Borel $\sigma$-algebra of the weak topology, i.e. $\mathcal{N}^{f}$ is the smallest $\sigma$-algebra with respect to which the evaluation $\boldsymbol{x} \mapsto n\left(\boldsymbol{x}_{B}\right)$ is measurable for every (bounded) Borel set $B \subseteq \mathcal{X}$.

Given a totally finite, non-atomic measure $\mu$ on $\mathcal{X}$, construct the Poisson process of intensity $\mu$ (typically $\mu$ is the restriction of Lebesgue measure to a compact window $A \subseteq \mathbb{R}^{d}$, yielding the unit rate Poisson process restricted to $A$ ). Let $\pi$ be its probability distribution on $\left(\Re^{f}, \mathcal{N}^{f}\right)$. Then we construct (Gibbs) point processes by specifying their density with respect to $\pi$. A density is a measurable function $p: \Re^{f} \rightarrow[0, \infty)$ that is integrable with respect to $\pi$. 
The general pairwise interaction process on a compact region $A \subseteq \mathbb{R}^{d}$ is then defined by its density

$$
p(\boldsymbol{x})=a \prod_{i} b\left(x_{i}\right) \prod_{i<j} c\left(x_{i}, x_{j}\right)
$$

(with respect to the unit-rate Poisson process on $A$ ) where $b, c$ are nonnegative measurable functions and $a$ is the normalising constant. The Strauss process (1.2) is the special case where $b(\cdot) \equiv \beta$ and $c(u, v)=\gamma$ if $0<\|u-v\| \leq r, c(u, v)=1$ otherwise. Kelly and Ripley (1976) pointed out that (1.2) is not integrable for $\gamma>1$.

\subsection{Area-interaction process}

Definition 1. (Standard case) The area-interaction process in a compact region $A \subseteq \mathbb{R}^{d}$ is the process with density

$$
p(\boldsymbol{x})=\alpha \beta^{n(\boldsymbol{x})} \gamma^{-m\left(U_{r}(\boldsymbol{x})\right)}
$$

with respect to the unit rate Poisson process on $A$, where $\beta, \gamma, r>0$ are parameters and $\alpha$ is the normalising constant, $m$ is Lebesgue measure, and

$$
U_{r}(\boldsymbol{x})=\bigcup_{i=1}^{n} B\left(x_{i}, r\right)
$$

is the union of spheres or discs of radius $r$ centred at the points of the realisation, $B\left(x_{i}, r\right)=\left\{a \in \mathbb{R}^{d}:\left\|a-x_{i}\right\| \leq r\right\}$.

For $\gamma=1$ this of course reduces to a Poisson process with intensity $\beta \mu$. It is intuitively clear that for $0<\gamma<1$ the pattern will tend to be 'ordered' and for $\gamma>1$ 'clustered' (we make this precise in Subsections 5.1 and 5.3). The clustered case was introduced by Widom and Rowlinson (1970). See also Hammersley et al. (1975) or Rowlinson (1980, 1990).

Various modifications are of interest, for example, one may wish to replace $U_{r}(\boldsymbol{x})$ by $A \cap U_{r}(\boldsymbol{x})$, or to allow the radii of the discs $B\left(x_{i}, r\right)$ to vary across the region (Lawson (1993)). More generally, the discs $B\left(x_{i}, r\right)$ can be replaced by compact sets $Z\left(x_{i}\right)$ depending on $x_{i}$. We assume that the mapping $Z$ onto the space $\mathcal{K}$ of all compact subsets is continuous with respect to the myopic topology (Matheron (1975), p. 12) generated by $\{K \in \mathcal{K}: K \cap F=\emptyset\}$ for all closed $F \subseteq \mathcal{X}$ and $\{K \in \mathcal{K}: K \cap G \neq \emptyset\}$ for all open subsets $G \subseteq \mathcal{X}$.

Definition 2. (General case) Let $\nu$ be a totally finite, Borel regular measure on $\mathcal{X}$ and $Z: \mathcal{X} \rightarrow \mathcal{K}$ a myopically continuous function, assigning to each point $a \in \mathcal{X}$ a compact set $Z(a) \subseteq \mathcal{X}$. Then the general area-interaction process is defined to have density

$$
p(\boldsymbol{x})=\alpha \beta^{n(\boldsymbol{x})} \gamma^{-\nu(U(x))}
$$


with respect to $\pi$ (the distribution of the finite Poisson process with intensity $\mu$ ), where $U(\boldsymbol{x})$ is the compact set $\bigcup_{i=1}^{n} Z\left(x_{i}\right)$.

In a parametric statistical model the measure $\nu$ and the definition of $Z(\cdot)$ might also be allowed to depend on the parameter $\theta$.

LEMMA 2.1. The density (2.2) is measurable and integrable for all fixed values of $\beta, \gamma>0$.

Proof. Let $t>0$ and consider $V=\left\{x \in \Re^{f}: \nu(U(x))<t\right\}$. We show that $V$ is open in the weak topology.

Choose $x \in V$. Since $\nu$ is regular, there is an open set $G \subseteq \mathcal{X}$ containing $U(x)$ such that $\nu(G)<t$. Consider $W=\left\{\boldsymbol{y} \in \Re^{f}: U(\boldsymbol{y}) \subseteq G\right\}$; we have $\boldsymbol{y} \in W$ iff $\boldsymbol{y}$ has no points in $H=\left\{a \in \mathcal{X}: Z(a) \cap G^{c} \neq \emptyset\right\}$. Now $H$ is closed in $\mathcal{X}$ since $a \mapsto Z(a)$ is myopically continuous and the class of all compact sets intersecting a given closed set is closed in the myopic topology on $\mathcal{K}$. Thus $W=\left\{\boldsymbol{y} \in \Re^{f}: n\left(\boldsymbol{y}_{H}\right)=0\right\}$ is open in the weak topology. But $\boldsymbol{x} \in W \subseteq V$ and $\boldsymbol{x}$ was arbitrary so $V$ is open in the weak topology.

In fact this shows that $\boldsymbol{x} \mapsto \nu(U(\boldsymbol{x}))$ is weakly upper semicontinuous. It follows that the map $g: \Re^{f} \rightarrow[0, \infty)$ defined by $\boldsymbol{x} \mapsto \exp [-\nu(U(\boldsymbol{x})) \log \gamma]$ is weakly upper semicontinuous for $\gamma \in(0,1)$ and lower semicontinuous for $\gamma>1$. Hence $g$ is measurable. By definition of the weak topology, $\boldsymbol{x} \mapsto \beta^{n(x)}$ is measurable, and hence the density $(2.2)$ is measurable.

To check integrability, observe that

$$
0 \leq \nu(U(\boldsymbol{x})) \leq \nu(\mathcal{X})<\infty
$$

Now the function $f(\boldsymbol{x})=\beta^{n(\boldsymbol{x})}$ is integrable, yielding the Poisson process with intensity measure $\beta \mu$. Hence (2.2) is dominated by an integrable function, hence integrable.

In fact (2.3) establishes a slightly stronger result.

LEMma 2.2. The distribution $P_{\beta, \gamma}$ of the general area interaction process is uniformly absolutely continuous with respect to the distribution of the Poisson process $\pi^{\beta}$ with intensity $\beta \mu$, that is its Radon-Nikodym derivative is uniformly bounded in $\boldsymbol{x}$.

In particular, the general area-interaction model satisfies the linear stability condition in Gates and Westcott (1986). Explicit bounds on the density $f$ with respect to a Poisson process with intensity $\beta \mu$ are

$$
\min \left\{\gamma^{\nu(\mathcal{X})}, \gamma^{-\nu(\mathcal{X})}\right\} \leq f \leq \max \left\{\gamma^{\nu(\mathcal{X})}, \gamma^{-\nu(\mathcal{X})}\right\} .
$$

This suggests that the 'singularity' (highly clustered behavior) of the Strauss model is unlikely for moderate values of $\gamma$. 
As usual, the normalising constant $\alpha$ is difficult to compute, since

$$
\alpha^{-1}=\mathbb{E} \beta^{n(X)} \gamma^{-\nu(U(X))}
$$

where the expectation is with respect to the reference Poisson process; this entails computing the moment generating function of $\nu(U(X))$, or equivalently, the vacancy distribution in the coverage problem (Hall (1988)). A notable exception is the 1-dimensional penetrable sphere model of Widom and Rowlinson (1970).

\subsection{Markov property}

The purpose of this paragraph is to place (2.2) in the context of Markov point processes in the sense of Ripley and Kelly (1977), see (Baddeley and Møller (1989), Kendall (1990)). Their defining property is that the likelihood ratio $\frac{p(x \cup\{a\})}{p(x)}$ for adding a new point $a$ to a configuration $x$ depends only on those $x_{i} \in x$ that are 'close' to $a$. Surveys can be found in Cressie ((1991), pp. 673-689), Stoyan et al. ((1987), pp. 148-166) and Stoyan and Stoyan ((1992), pp. 342-359).

As in Baddeley and Van Lieshout $(1991,1992,1993)$ define two points $a, b \in \mathcal{X}$ to be neighbours (and write $a \sim b$ ) whenever $Z(a) \cap Z(b) \neq \emptyset$. In the standard case $a \sim b$ iff $\|a-b\| \leq 2 r$.

LEMMA 2.3. The area-interaction process (2.2) is a Markov point process with respect to $\sim$ in the sense of Ripley and Kelly (1977).

Proof. The likelihood ratio

$$
\frac{p(\boldsymbol{x} \cup\{a\})}{p(\boldsymbol{x})}=\beta \gamma^{-\nu(Z(a) \backslash U(x))}
$$

is computable in terms of $a$ and $\left\{x_{i}: x_{i} \sim a\right\}$, since

$$
\begin{aligned}
Z(a) \backslash U(\boldsymbol{x}) & =Z(a) \cap\left[\bigcup_{i} Z\left(x_{i}\right)\right]^{c} \\
& =Z(a) \cap\left[\bigcup_{x_{i} \sim a} Z\left(x_{i}\right)\right]^{c} .
\end{aligned}
$$

Hence (2.2) defines a Markov point process with respect to $\sim$

The Ripley-Kelly analogue of the Hammersley-Clifford theorem (Ripley and Kelly (1977)) then implies that the density $p$ can be written as a product of clique
interaction terms

$$
p(\boldsymbol{x})=\prod_{\boldsymbol{y} \subseteq x} q(\boldsymbol{y})
$$

where $q(\boldsymbol{y})=1$ unless $y_{i} \sim y_{j}$ for all elements of $y$. To compute the interaction terms explicitly, invoke the inclusion-exclusion formula:

$$
\begin{aligned}
\nu(U(\boldsymbol{x}))= & \sum_{i=1}^{n} \nu\left(Z\left(x_{i}\right)\right) \\
& -\sum_{i<j} \nu\left(Z\left(x_{i}\right) \cap Z\left(x_{j}\right)\right)+\cdots+(-1)^{n+1} \nu\left(\bigcap_{i=1}^{n} Z\left(x_{i}\right)\right)
\end{aligned}
$$


which gives

$$
\begin{aligned}
& q(\emptyset)=\alpha \\
& q(\{a\})=\beta \gamma^{-\nu(Z(a))} \\
& q\left(\left\{y_{1}, \ldots, y_{k}\right\}\right)=\gamma^{(-1)^{k} \nu\left(\bigcap_{i=1}^{k} Z\left(y_{i}\right)\right)}, \quad k \geq 2 .
\end{aligned}
$$

That is, the process exhibits interactions of infinite order.

It is also trivial to verify that the process satisfies a spatial Markov property (cf. Kendall (1990), Ripley and Kelly (1977)). Define the dilation of a set $E \subseteq \mathcal{X}$ by

$$
D_{Z}(E)=\{u \in \mathcal{X}: \exists e \in E \text { such that } Z(u) \cap Z(e) \neq \emptyset\} ;
$$

in the standard case this becomes the classical dilation of mathematical morphology

$$
D_{Z}(E)=\left\{u \in \mathbb{R}^{d}: d(u, E) \leq 2 r\right\}
$$

where $d(u, E)=\inf \{\|u-v\|: v \in E\}$. Then the spatial Markov property states that the restriction of the process to $E$ is conditionally independent of the restriction to $D_{Z}(E)^{c}$ given the information in $D_{Z}(E) \backslash E$.

\subsection{Limiting cases}

Here we study the convergence of the area-interaction process as $\gamma \rightarrow 0, \infty$.

Let $\nu^{*}=\max _{x} \nu(U(\boldsymbol{x}))$, typically the measure of the observation window or its (generalised) dilation and

$$
H=\left\{\boldsymbol{x}: \nu(U(\boldsymbol{x}))=\nu^{*}\right\} .
$$

Further let $\pi^{\beta}$ be the distribution of the Poisson process of rate $\beta$ in $A$ and finally, in the standard case, denote

$$
H C=\left\{\boldsymbol{x}: m(U(\boldsymbol{x}))=n(\boldsymbol{x}) \pi r^{2}\right\} .
$$

LEMMA 2.4. Let $P_{\beta, \gamma}$ be the distribution of the area interaction process with density (2.2).

If $\gamma \rightarrow 0$ with $\beta$ fixed, then $P_{\beta, \gamma}$ converges to a uniform process on $H$, i.e. $P_{\beta, \gamma}(E) \rightarrow \pi^{\beta}(E \cap H) / \pi^{\beta}(H)$.

In the standard case, if $\gamma \rightarrow 0$ and $\beta \rightarrow 0$ so that $\beta \gamma^{-\pi r^{2}} \rightarrow \zeta \in(0, \infty)$, then $P_{\beta, \gamma}$ converges to $P(E)=\pi^{\zeta}(E \cap H C) / \pi^{\zeta}(H C)$, a hard core process.

If $\gamma \rightarrow \infty$ with $\beta<\infty$ fixed, then $P_{\beta, \gamma}$ converges to a process that is empty with probability 1 .

Proof. First consider $\gamma \rightarrow 0$. Then $\int \gamma^{\nu^{*}-\nu(U(x))} d \pi^{\beta}(\boldsymbol{x}) \rightarrow \pi^{\beta}(H)$, hence

$$
p_{\beta, \gamma}(\boldsymbol{x})=\frac{\gamma^{\nu^{*}-\nu(U(x))}}{\int \gamma^{\nu^{*}-\nu(U(x))} d \pi^{\beta}(\boldsymbol{x})} \rightarrow \frac{\mathbf{1}\{\boldsymbol{x} \in H\}}{\pi^{\beta}(H)}
$$


from which the first assertion follows. To prove the second assertion, note that for $m\left(U_{r}(\boldsymbol{x})\right)<n(\boldsymbol{x}) \pi r^{2}, \gamma^{n \pi r^{2}-m\left(U_{r}(x)\right)} \rightarrow 0$. Hence

$$
p_{\beta, \gamma}(x) \rightarrow \frac{\zeta^{n(x)} \mathbf{1}\{\boldsymbol{x} \in H C\}}{\int_{H C} \zeta^{n(x)} d \pi^{1}(x)} .
$$

The third statement follows similarly, by noting that the density converges pointwise to zero unless the pattern is empty.

\subsection{Interpretation and motivation}

Area-interaction seems a plausible model for some biological processes. For example the points $x_{i}$ may represent plants or animals which consume food within a radius $r$ of their current location. The total area of accessible food is then $U(\boldsymbol{x})$, and the herd will tend to maximise this area, so an area-interaction model with $\gamma<1$ is plausible. Alternatively assume that the animals or plants are hunted by a predator which appears at a random position and catches any prey within a distance $r$. Then $U(x)$ is the area of vulnerability, and the herd as a whole should tend to minimise this (see, e.g. Hamilton (1971)) so an area-interaction model with $\gamma>1$ is plausible.

The following trivial interpretations are also possible:

Lemma 2.5. Let $X, Y$ be independent Poisson processes in $A$ with intensity measures $\beta \mu$ and $|\log \gamma| \nu$ respectively. If $\gamma>1$ then the conditional distribution of $X$ given $\{Y \cap U(X)=\emptyset\}$ is an area-interaction process with parameter $\gamma$. If $\gamma<1$ then the conditional distribution of $X$ given $\{Y \subseteq U(X)\}$ is an area-interaction process with parameter $\gamma$.

ProOF. If $\gamma>1$

$$
\mathbb{P}\{Y \cap U(X)=\emptyset \mid X\}=e^{-\nu(U(X)) \log \gamma}=\gamma^{-\nu(U(X))}
$$

hence the conditional distribution of $X$ given $Y \cap U(X)=\emptyset$ has a density proportional to the right hand side. Similarly if $\gamma<1$

$$
\begin{aligned}
\mathbb{P}\{Y \subseteq U(X) \mid X\} & =\mathbb{P}\{Y \cap(A \backslash U(X))=\emptyset\} \\
& =e^{\nu(A \backslash U(X)) \log \gamma}=\gamma^{\nu(A)} \gamma^{-\nu(U(X))} .
\end{aligned}
$$

Other interpretations are available in terms of spatial birth-and-death processes (see Section 4). Briefly, if the points represent plants again, we may consider a process in which existing plants have exponential(1) lifetimes, and a new seed takes root at location $a$ with rate $p(\boldsymbol{x} \cup\{a\}) / p(\boldsymbol{x})$ related to the area accessible to $a$ that is not already accessible to an existing plant. Alternatively we may assume the plants or animals arrive at a constant rate uniformly over space, and an existing animal $x_{i}$ dies at a rate $p\left(\boldsymbol{x} \backslash\left\{x_{i}\right\}\right) / p(\boldsymbol{x})$ related to the risk of being
attacked by a predator. 


\section{Stationary area-interaction process}

Here we use the methods of Preston (1976) to check that the area interaction model (standard case) can be considered as the restriction to a bounded sampling window of a stationary point process on the whole of $\mathbb{R}^{d}$.

Let $\Re$ be the space of all locally finite counting measures (i.e. integer-valued Radon measures) on $\mathbb{R}^{d}$ with the vague topology, and $\mathcal{N}$ its Borel $\sigma$-algebra; that is, $\mathcal{N}$ is the smallest $\sigma$-algebra making $\boldsymbol{x} \mapsto n\left(\boldsymbol{x}_{B}\right)$ measurable for all bounded Borel sets $B$.

Write $\mathcal{C}$ for the class of all bounded Borel sets in $\mathbb{R}^{d}$. For every $B \in \mathcal{C}$ let $\Re_{B}$ be the subspace of those $x \in \Re$ contained in $B$ (i.e. putting no mass outside $B), \mathcal{N}_{B} \subseteq \mathcal{N}$ the induced $\sigma$-algebra on $\Re$ and $\pi_{B}^{\beta}$ the distribution on $(\Re, \mathcal{N})$ of the homogeneous Poisson process of rate $\beta$ on $B$. Note that any $\boldsymbol{x} \in \Re$ can be decomposed as $x=x_{B} \cup x_{B^{c}}$. Define $f^{B}: \Re \rightarrow[0, \infty)$ by

$$
f^{B}(\boldsymbol{x})=\alpha_{B}\left(\boldsymbol{x}_{B^{c}}\right) \gamma^{-m\left(U_{r}(\boldsymbol{x}) \cap B_{\oplus r}\right)}
$$

where $U_{r}(\boldsymbol{x})=\bigcup_{x_{i} \in x} B\left(x_{i}, r\right), B_{\oplus r}$ the dilation of $B$ by a ball with radius $r$ and $\alpha_{B}\left(x_{B^{c}}\right)$ is the normalising constant

$$
\alpha_{B}\left(\boldsymbol{x}_{B^{c}}\right)^{-1}=\int_{\Re_{B}} \gamma^{-m\left(U_{r}\left(\boldsymbol{y} \cup x_{B^{c}}\right) \cap B_{\oplus r}\right)} d \pi_{B}^{\beta}(\boldsymbol{y}) .
$$

To check that (3.1) is measurable and integrable, observe that

$$
U_{r}(\boldsymbol{x}) \cap B_{\oplus r}=U_{r}\left(\boldsymbol{x}_{B_{\oplus 2 r}}\right) \cap B_{\oplus r}
$$

so that the map $g: \boldsymbol{x} \mapsto m\left(U_{r}(\boldsymbol{x}) \cap B_{\oplus r}\right)$ is measurable with respect to $\mathcal{N}_{B_{\oplus 2 r}}$ and a fortiori with respect to $\mathcal{N}$. Clearly $g$ is integrable with respect to $\pi_{B_{\oplus 2 r}}^{\beta}$. Regarding the Poisson process on $B_{\oplus 2 r}$ as the independent superposition of Poisson processes on $B$ and $B_{\oplus 2 r} \backslash B$ we can apply Fubini's theorem to integrate over the $B$ component and conclude that $\alpha_{B}(\cdot)^{-1}$ is $\mathcal{N}_{B_{\oplus 2 r} \backslash B^{-} \text {-measurable and integrable. }}$ Hence, (3.1) is $\mathcal{N}$-measurable and integrable, and we may define for $\boldsymbol{x} \in \Re, F \in \mathcal{N}$

$$
\kappa_{B}(\boldsymbol{x}, F)=\int_{\Re_{B}} 1_{F}\left(\boldsymbol{x}_{B^{c}} \cup \boldsymbol{y}\right) f^{B}\left(\boldsymbol{x}_{B^{c}} \cup \boldsymbol{y}\right) d \pi_{B}^{\beta}(\boldsymbol{y}) .
$$

THEOREM 3.1. There exists a stationary point process $X$ on $\mathbb{R}^{d}$ such that

$$
\mathbb{P}\left\{X \in F \mid X_{B^{c}}\right\}=\kappa_{B}(X, F) \quad \text { a.s. }
$$

for all $B \in \mathcal{C}$ and $F \in \mathcal{N}$. That is, (3.2) is a specification without forbidden states (Preston (1976), p. 12) and the distribution of $X$ is a stationary Gibbs state with this specification. The corresponding potential $V: \Re^{f} \rightarrow \mathbb{R}$,

$$
V(\boldsymbol{x})=(-\log \gamma) m(U(\boldsymbol{x}))
$$


is stable (Preston (1976), p. 96).

Note that this result does not exclude the possibility that the Gibbs state is not unique, i.e. there may be 'phase transition' (Preston (1976), p. 46).

Proof. First we prove consistency condition (6.10) of Preston ((1976), p. 91). For any bounded Borel sets $A \subseteq B$

$$
\frac{f^{B}(\boldsymbol{x})}{f^{A}(\boldsymbol{x})}=\frac{\alpha_{B}\left(\boldsymbol{x}_{B^{c}}\right)}{\alpha_{A}\left(\boldsymbol{x}_{A^{c}}\right)} \gamma^{-m\left(U_{r}\left(\boldsymbol{x}_{A^{c}}\right) \cap B_{\oplus r} \backslash A_{\oplus r}\right)} .
$$

On the other hand

$$
\int_{\Re_{A}} f^{B}\left(\boldsymbol{x}_{\boldsymbol{A}^{c}} \cup \boldsymbol{y}\right) d \pi_{A}^{\beta}(\boldsymbol{y})=\alpha_{B}\left(\boldsymbol{x}_{B^{c}}\right) \int_{\Re_{A}} \gamma^{-m\left(U_{r}\left(x_{A^{c}} \cup \boldsymbol{y}\right) \cap B_{\oplus r}\right)} d \pi_{A}^{\beta}(\boldsymbol{y}) .
$$

Since

$$
\begin{aligned}
m\left(U_{r}\left(\boldsymbol{x}_{A^{c}} \cup \boldsymbol{y}\right) \cap B_{\oplus r}\right)= & m\left(U_{r}\left(\boldsymbol{x}_{A^{c}} \cup \boldsymbol{y}\right) \cap A_{\oplus r}\right) \\
& +m\left(U_{r}\left(\boldsymbol{x}_{A^{c}}\right) \cap B_{\oplus r} \backslash A_{\oplus r}\right),
\end{aligned}
$$

$\int_{\Re_{A}} f^{B}\left(\boldsymbol{x}_{A^{c}} \cup \boldsymbol{y}\right) d \pi_{A}^{\beta}(\boldsymbol{y})=f^{B}(\boldsymbol{x}) / f^{A}(\boldsymbol{x})$. It follows (Preston (1976), pp. 90-91) that $\left\{\kappa_{B}: B \in \mathcal{C}\right\}$ is a specification in the sense of (Preston (1976), p. 12).

Now we check the conditions of Theorem 4.3 of Preston ((1976), p. 58). Condition (3.6) of Preston ((1976), p. 35) is trivially satisfied. Arguments similar to those used to derive Lemma 2.2 above yield that for any $K \in \mathcal{K}$ the family

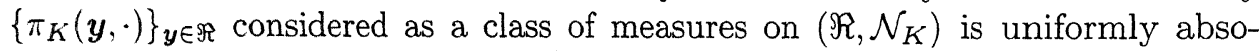
lutely continuous with respect to $\pi_{K}^{\beta}$; hence Preston's condition (3.11) (Preston (1976), p. 41) holds, which implies his (3.10). It remains to check (3.8) of Preston ((1976), p. 35). Let $\mathcal{K}$ be the class of all compact subsets of $\mathbb{R}^{d}$; then we claim that

for any $B \in \mathcal{C}$ and $F \in \mathcal{N}_{K}$ where $K \in \mathcal{K}$, there exists $L \in \mathcal{K}$ such that $\kappa_{B}(\cdot, F)$ is measurable with respect to $\mathcal{N}_{L}$.

To check this, choose $L$ to contain $K \cup B_{\oplus 2 r}$ and observe that $1_{F}\left(x_{B^{c}} \cup y\right)$ and $f^{B}\left(\boldsymbol{x}_{B}^{c} \cup \boldsymbol{y}\right)$ are measurable with respect to $\mathcal{N}_{L} \otimes \mathcal{N}_{B}$, then apply Fubini's theorem (in the same way as was used to prove measurability of (3.1)). This proves the claim, which implies Preston's (3.8) and hence the conditions of his Theorem 4.3.

It is easy to see that $V$ is the unique canonical potential corresponding to our densities $f^{\mathcal{B}}$ (cf. Preston (1976), p. 92). Since $0 \leq m\left(U_{r}(\boldsymbol{x})\right) \leq \pi r^{2} n(\boldsymbol{x}), V$ is stable. 


\section{Simulation}

In this section we explore methods for simulating (2.1) using spatial birthand-death processes (Baddeley and Møller (1989), Møller (1989), Preston (1977)). Other techniques such as Metropolis-Hastings algorithms (Geyer and Møller (1993), Møller (1992)) are clearly possible. The general case can be dealt with similarly, but for simplicity we focus on the standard case.

A spatial birth-and-death process (Preston (1977)) is a continuous time, pure jump Markov process with states in $\Re^{f}$ specified by its transition rates $D(\boldsymbol{x})$ $\left\{x_{i}\right\}, x_{i}$ ) for a death (transition from $\boldsymbol{x}$ to $\left.\boldsymbol{x} \backslash\left\{x_{i}\right\}\right)$ and $b(\boldsymbol{x}, u) d \mu(u)$ for a birth (transition from $\boldsymbol{x}$ to $\boldsymbol{x} \cup\{u\}$ ). Write $D(\boldsymbol{x})=\sum_{x_{i} \in x} D\left(\boldsymbol{x} \backslash\left\{x_{i}\right\}, x_{i}\right)$ and $B(\boldsymbol{x})=$ $\int_{A} b(\boldsymbol{x}, u) d \mu(u)$ for the total transition rates out of state $\boldsymbol{x}$.

We consider two standard cases, the constant death rate process (Ripley (1977)) which has death rate $D\left(\boldsymbol{x} \backslash\left\{x_{i}\right\}, x_{i}\right)=1$ and birth rate

$$
b(\boldsymbol{x}, u)=\frac{p(\boldsymbol{x} \cup\{u\})}{p(\boldsymbol{x})}=\beta \exp [-\log (\gamma) \nu(U(\boldsymbol{x} \cup\{u\}) \backslash U(\boldsymbol{x}))]
$$

and the constant birth rate process which has $b(\boldsymbol{x}, u) \equiv 1$ and

$$
D\left(\boldsymbol{x} \backslash\left\{x_{i}\right\}, x_{i}\right)=\frac{p\left(\boldsymbol{x} \backslash\left\{x_{i}\right\}\right)}{p(\boldsymbol{x})}=\beta^{-1} \exp \left[\log (\gamma) \nu\left(U(\boldsymbol{x}) \backslash U\left(\boldsymbol{x} \backslash\left\{x_{i}\right\}\right)\right)\right] .
$$

LEMMA 4.1. For any $\gamma$, the constant death rate and constant birth rate processes exist and converge in distribution to the area-interaction process (2.1) from any initial state.

Proof. Note that $p(\boldsymbol{x})>0$ for all $\boldsymbol{x}$. Hence we only have to check the summability condition of Theorem 2.10 in Baddeley and Møller (1989) (see Proposition 5.1, Theorem 7.1 in Preston (1977)). These are obviously satisfied since the birth rates are bounded by a constant times $\beta^{n(x)}$.

On a practical note, computation of the ratios (4.1) or (4.2) at every $u$ is equivalent to computing the Hough transform of $U(\boldsymbol{x})$; see e.g. Baddeley and Van Lieshout (1992) or Illingworth and Kittler (1988). The fixed $n$, alternating birth/death technique of Ripley (1977) was used to generate Fig. 1. This requires that we generate a point $u \in A$ with density proportional to (4.1); this is relatively easy using rejection sampling, since (4.1) is dominated by a known constant by virtue of $(2.3)$ which in practice will be a good bound when $\gamma$ is not far from 1 .

\section{Inference}

\subsection{Sufficient statistics, exponential families}

Consider a family of area-interaction processes (2.1) indexed by parameter $\theta=(\beta, \gamma)$, with $r$ and $A \subseteq \mathbb{R}^{d}$ fixed. This is an exponential family with canonical sufficient statistic

$$
T(\boldsymbol{x})=(n(\boldsymbol{x}), M(\boldsymbol{x}, \cdot))
$$


where

$$
M(\boldsymbol{x}, r)=m\left(U_{r}(\boldsymbol{x})\right)=m\left(\bigcup_{i=1}^{n} B\left(x_{i}, r\right)\right) .
$$

Modifying this slightly we obtain a connection with the 'empty space statistic' $F(t)=\mathbb{P}\{X \cap B(0, t) \neq \emptyset\}$ (Diggle (1983), Ripley (1981)). Define

$$
\hat{F}(r)=\frac{m\left(A_{(-r)} \cap\left(\bigcup_{i=1}^{n} B\left(x_{i}, r\right)\right)\right)}{m\left(A_{(-r)}\right)}
$$

where

$$
A_{(-t)}=\{a \in A: B(a, t) \subseteq A\} .
$$

This is the 'border method' (Ripley (1988), p. 25) or 'reduced sample' estimator (Baddeley and Gill (1992)) of the empty space function.

LEMMA 5.1. $(n(\boldsymbol{x}), \hat{F})$ is a sufficient statistic for the area-interaction process (2.2) with parameters $(\beta, \gamma)$ when $Z(a)=B(a, r)$ and the measure $\nu$ is Lebesgue measure restricted to $A_{(-r)}$.

The canonical parameter is $\theta=-\log \gamma$ but we prefer to use $\gamma$ to maintain the comparison with the Strauss process.

\subsection{Maximum likelihood}

As usual for Markov point processes, the likelihood (2.2) is easy to compute except for a normalising constant $\alpha$ that is not known analytically. Maximum likelihood estimation therefore rests on numerical or Monte Carlo approximations of $\alpha$ (Ogata and Tanemura $(1981,1984,1989)$, Penttinen (1984)) or recursive approximation methods (Moyeed and Baddeley (1991)). For a more detailed review see Diggle et al. (1994), Ripley (1988) or Geyer and Thompson (1992).

We will not explore this further here, except to note that the maximum likelihood estimating equations are as usual

$$
\begin{aligned}
& n(\boldsymbol{x})=\mathbb{E}_{\beta, \gamma} n(X) \\
& \nu(U(\boldsymbol{x}))=\mathbb{E}_{\beta, \gamma} \nu(U(X))
\end{aligned}
$$

where $\boldsymbol{x}$ is the observed pattern and $X$ is a random pattern with density (2.2). For the model conditioned on $n(\boldsymbol{x})=n$ the ML estimating equation is analogous to (5.3) with $\beta$ absent.

Other estimation techniques have been proposed in Diggle et al. (1987) and Diggle and Gratton (1984). 


\subsection{Takacs-Fiksel}

The Takacs-Fiksel estimation method exploits the Nguyen-Zessin identity (Nguyen and Zessin (1979))

$$
\lambda \mathbb{E}_{a}^{!} f(X)=\mathbb{E}[\lambda(a ; X) f(X)]
$$

holding for any bounded measurable non-negative function $f: \Re \rightarrow \mathbb{R}_{+}$and any stationary Gibbs process $X$ on $\mathbb{R}^{d}$ with finite intensity $\lambda$, see Glötz $(1980 a, 1980 b)$, Kozlov (1976), Matthes et al. (1979), Kallenberg (1984) or Ripley ((1988), pp. 54$55)$, Diggle et al. $((1994), \S 2.4)$. The expectation on the left side of (5.4) is with respect to the reduced Palm distribution of $X$ at $a \in \mathbb{R}^{d}$, and $\lambda(a ; \boldsymbol{x})$ is the Papangelou conditional intensity of $X$ at $a$. The idea (Fiksel $(1984,1988)$, Takacs $(1983,1986))$ is then to choose suitable functions and estimate both sides in the above formula. The resulting set of equations is solved, yielding estimates for the parameters of the model.

When $X$ is a Gibbs point process the conditional intensity can be computed in terms of the potential (Kallenberg (1984)). For the standard area-interaction process the conditional intensity is

$$
\lambda(u ; \boldsymbol{x})=\frac{p(\boldsymbol{x} \cup\{u\})}{p(\boldsymbol{x} \backslash\{u\})}=\beta \gamma^{-m\left(B(u, r) \backslash U_{r}(\boldsymbol{x} \backslash\{u\})\right)} .
$$

In case $u \notin \boldsymbol{x}$ this reduces to

$$
\beta \gamma^{-m\left(B(u, r) \backslash U_{r}(x)\right)} .
$$

One interesting instance of (5.4) is

$$
f(\boldsymbol{x})=\frac{\mathbf{1}\{\boldsymbol{x} \cap B(0, s)=\emptyset\}}{\lambda(0 ; \boldsymbol{x})}
$$

using 0 as an arbitrary point of $\mathbb{R}^{d}$ (cf. Stoyan et al. (1987), (5.5.18), p. 159). Then $\mathbb{E}[\lambda(0 ; X) f(X)]=1-F(s)$ where $F(s)=\mathbb{P}\{X \cap B(0, s) \neq \emptyset\}$ is the empty space function of $X$. Now if $s>2 r$ then

$$
\begin{aligned}
\lambda \mathbb{E}_{0}^{!} f(X) & =\lambda \int \mathbf{1}\{X \cap B(0, s)=\emptyset\} \beta^{-1} \gamma^{m\left(B(0, r) \backslash U_{r}(X)\right)} d P_{0}^{!}(X) \\
& =\lambda \int 1\{X \cap B(0, s)=\emptyset\} \beta^{-1} \gamma^{m(B(0, r))} d P_{0}^{!}(\boldsymbol{x}) \\
& =\lambda \beta^{-1} \gamma^{\pi r^{2}}[1-G(s)]
\end{aligned}
$$

where $G(s)=\mathbb{P}_{0}^{!}\{X \cap B(0, s) \neq \emptyset\}$ is the nearest neighbour distance distribution function of $X$. Equivalently,

$$
\lambda \frac{1-G(s)}{1-F(s)}=\beta \gamma^{-\pi r^{2}}
$$


for all $s>2 r$. This suggests that parameter estimates for the area-interaction process can be extracted directly from the standard statistics $F$ and $G$. For further development of this idea see Van Lieshout and Baddeley (1995).

The identity (5.6) provides a further description of the typical pattern generated by an area-interaction process, since the ratio $(1-G) /(1-F)$ is less than 1 for clustered patterns, 1 for a Poisson process and greater than 1 for regular patterns.

\subsection{Pseudolikelihood estimation}

The pseudolikelihood is defined (Besag (1977), Jensen and Møller (1991)) by

$$
P L(\beta, \gamma ; \boldsymbol{x})=\exp \left\{-\int_{A} \lambda(u ; \boldsymbol{x}) d u\right\} \prod_{i=1}^{n} \lambda\left(x_{i} ; \boldsymbol{x}\right)
$$

and can be interpreted as the limit case of pseudolikelihood for lattice processes (Besag (1977), Besag et al. (1982), Jensen and Møller (1991)). For Markov processes of finite range, maximum pseudolikelihood estimators are consistent (Jensen and Møller (1991)); asymptotic normality is considered in Jensen (1993).

For the area-interaction model, the maximum pseudolikelihood estimates of $\beta$ and $\gamma$ are the solutions of

$$
\begin{aligned}
& n=\beta \int_{A} \gamma^{-t(u)} d u \\
& \sum_{i=1}^{n} t\left(x_{i}\right)=\beta \int_{A} t(u) \gamma^{-t(u)} d u
\end{aligned}
$$

where $t(u)=m\left(B(u, r) \backslash U_{r}(\boldsymbol{x} \backslash\{u\})\right)$.

Note that these have exactly the same form as the pseudolikelihood equations for the Strauss model (Ripley (1988), p. 53); in that case $-t(u)$ is the number of points in $\boldsymbol{x}$ with $0<\left\|u-x_{i}\right\| \leq r$.

For inhibitory pairwise interaction models it is known that pseudolikelihood estimation is a special case of the Takacs-Fiksel method when the interaction radius $r$ is fixed (Diggle et al. ((1994), Section 2.4), Ripley ((1988), p. 54), Särkkä ((1990), Section 4)). The same is true for the area-interaction model.

THEOREM 5.1. For a stationary area-interaction process, the pseudolikelihood equations (5.8) and (5.9) are special cases of the Takacs-Fiksel method.

Proof. Take $f$ to be either of

$$
\begin{aligned}
& f_{\beta}(x)=\beta^{-1} \\
& f_{\gamma}(\boldsymbol{x})=-\gamma^{-1} m\left(B(0, r) \backslash U_{r}(\boldsymbol{x} \backslash\{0\})\right) .
\end{aligned}
$$

These are the partial derivatives of $\log \lambda(0 ; \boldsymbol{x})$ with respect to $\beta$ and $\gamma$. When $f=f_{\beta}$ an unbiased estimator for the left hand side of (5.4) is

$$
\frac{n}{m(A)} \frac{1}{\beta}
$$


and, by stationarity,

$$
\frac{1}{m(A)} \int_{A} \frac{1}{\beta} \beta \gamma^{-t(u)} d u
$$

is an unbiased estimator of the right hand side. When $f=f_{\gamma}$, the average over the observed events

$$
\frac{n}{m(A)} \frac{1}{n} \sum_{i=1}^{n} \frac{-t\left(x_{i}\right)}{\gamma}
$$

is an unbiased estimator of the left hand side of (5.4) by the Campbell-Mecke formula, while an unbiased estimator for the right hand side is the window mean

$$
\frac{1}{m(A)} \int_{A} \frac{-t(u)}{\gamma} \beta \gamma^{-t(u)} d u
$$

These reduce to (5.8)-(5.9).

\section{Approximation by lattice processes}

Besag et al. (1982) proved that any purely inhibitory (or hard core) pairwise interaction point process is the weak limit of a sequence of lattice processes, and remark that this is also true of general Gibbs point processes, again of purely inhibitory type. Here we prove a similar result for the area-interaction model, which is not purely inhibitory.

Consider a partition $\left\{C_{1}, \ldots, C_{m}\right\}$ of the observation window $A$ and choose fixed points $\xi_{i} \in C_{i}$. Denote the area of $C_{i}$ by $A_{i}>0$ and the set of all representatives by $\Xi$. We shall construct a 0,1 -valued stochastic process $\boldsymbol{n}=\left\{n_{i}: i=\right.$ $1, \ldots, m\}$ which is auto-logistic,

$$
\frac{\mathbb{P}\left(n_{i}=1 \mid n_{j}, j \neq i\right)}{\mathbb{P}\left(n_{i}=0 \mid n_{j}, j \neq i\right)}=\mu_{i}\left(C_{i}\right)
$$

where

$$
\mu_{i}\left(C_{i}\right)=A_{i} \prod q\left(\xi_{i} \cup \boldsymbol{y}\right)^{\eta(y)} ;
$$

the product ranges over all (possibly empty) subsets of $\Xi \backslash\left\{\xi_{i}\right\}$, the set function $q$ is the clique interaction function (2.5) of the area-interaction model, and $\eta(\boldsymbol{y})=$ $\prod_{\xi_{j} \in y} n_{j}$ is either 0 or 1 , defining $0^{0}=1$.

Given a realisation of $n$, construct a point process $x$ such that if $n_{i}=0$ then $\boldsymbol{x} \cap C_{i}$ is empty, while if $n_{i}=1$ then $\boldsymbol{x} \cap C_{i}$ consists of one point uniformly distributed in $C_{i}$ independently of other points.

LEMMA 6.1. The conditional distributions (6.1) specify a distribution for $\boldsymbol{n}$ of the form

$$
\frac{\boldsymbol{P}(\boldsymbol{n})}{\boldsymbol{P}(\mathbf{0})}=\prod_{i} A_{i}^{n_{i}} \prod_{\emptyset \neq y \subseteq \Xi} q(\boldsymbol{y})^{\eta(\boldsymbol{y})} .
$$


The point process $\boldsymbol{x}$ is absolutely continuous with respect to the unit rate Poisson process on $A$, with density

$$
f(\boldsymbol{x})=f(\emptyset) \prod_{\emptyset \neq y \subseteq \Xi} q(\boldsymbol{y})^{\eta_{x}(y)}
$$

Here $\eta_{x}(\boldsymbol{y})=\prod n\left(\boldsymbol{x} \cap C_{j}\right)$ and the product ranges over all $j$ such that $\xi_{j} \in \boldsymbol{y}$.

Proof. Use Besag's factorisation theorem (Besag (1974), p. 195).

THEOREM 6.1. Consider a sequence of partitions $\mathcal{C}_{r}=\left\{C_{r, 1}, \ldots, C_{r, m(r)}\right\}$ such that $\max _{i} \operatorname{diam}\left(C_{r, i}\right) \rightarrow 0$. Then the corresponding point process $\boldsymbol{x}^{(r)}$ converges weakly and in total variation to the area-interaction process.

Proof. Let $f_{r}$ be the density of $\boldsymbol{x}^{(r)}$. For fixed $\boldsymbol{x}$

$$
\frac{f_{r}(\boldsymbol{x})}{f_{r}(\emptyset)} \rightarrow \prod_{\emptyset \neq y \subseteq x} q(\boldsymbol{y})=\beta^{n(\boldsymbol{x})} \gamma^{-\nu(U(\boldsymbol{x}))}
$$

since all cells contain at most one point, and $q$ is continuous in all its arguments. By dominated convergence,

$$
\frac{1}{f_{r}(\emptyset)}=\int \frac{f_{r}(\boldsymbol{x})}{f_{r}(\emptyset)} d \pi(\boldsymbol{x}) \rightarrow \int \frac{1}{\alpha} p(\boldsymbol{x}) d \pi(\boldsymbol{x})=\frac{1}{\alpha}
$$

thus,

$$
f_{r}(\boldsymbol{x})=\frac{f_{r}(\boldsymbol{x})}{f_{r}(\emptyset)} f_{r}(\emptyset) \rightarrow p(\boldsymbol{x})
$$

pointwise and the theorem is proved.

\section{Final remarks}

The Strauss process is a special case of the general pairwise interaction process. In the same way, there is a generalisation of the area-interaction process to a process with density

$$
p(\boldsymbol{x})=a \exp \left(-\int_{A} f(d(\boldsymbol{x}, u)) d u\right)
$$

where $d(\boldsymbol{x}, u)=\min _{i}\left\|x_{i}-u\right\|$ and $f:[0, \infty] \rightarrow(-\infty, \infty]$. The area-interaction process is then the special case $f(t)=1[t \leq r]$. Thus $f$ is the analogue of the general interaction function in pairwise interaction. 


\section{Acknowledgements}

This research was carried out at CWI (both authors) and the Free University of Amsterdam (Van Lieshout). We thank Profs. Besag, Møller and Oosterhoff for helpful comments and Prof. Ogata and an anonymous referee for drawing our attention to the penetrable sphere model.

\section{REFERENCES}

Baddeley, A. J. and Gill, R. D. (1992). Kaplan-Meier estimators for interpoint distance distributions of spatial point processes, Research Report, 718, Mathematical Institute, University of Utrecht. The Netherlands.

Baddeley, A. J. and Møller, J. (1989). Nearest-neighbour Markov point processes and random sets, Internat. Statist. Rev., 57, 89-121.

Baddeley, A. J. and Van Lieshout, M. N. M. (1991). Recognition of overlapping objects using Markov spatial models, Tech. Report, BS-R9109, CWI, Amsterdam.

Baddeley, A. J. and Van Lieshout, M. N. M. (1992). ICM for object recognition, Computational Statistics (eds. Y. Dodge and J. Whittaker), Vol. 2, 271-286, Physica/Springer, Heidelberg.

Baddeley, A. J. and Van Lieshout, M. N. M. (1993). Stochastic geometry models in high-level vision, Statistics and Images (eds. K. Mardia and G. K. Kanji), Vol. 1, Journal of Applied Statistics, 20, 233-258, Carfax, Abingdon.

Besag, J. (1974). Spatial interaction and the statistical analysis of lattice systems (with discussion), J. Roy. Statist. Soc. Ser. B, 36, 192-236.

Besag, J. (1977). Some methods of statistical analysis for spatial data, Bull. Internat. Statist. Inst., 47, 77-92.

Besag, J., Milne, R. and Zachary, S. (1982). Point process limits of lattice processes, J. Appl. Probab., 19, 210-216.

Cressie, N. A. C. (1991). Statistics for Spatial Data, Wiley, New York.

Daley, D. J. and Vere-Jones, D. (1988). An Introduction to the Theory of Point Processes, Springer, New York.

Diggle, P. J. (1983). Statistical Analysis of Spatial Point Patterns, Academic Press, London.

Diggle, P. J. and Gratton, R. J. (1984). Monte Carlo methods of inference for implicit statistical models, J. Roy. Statist. Soc. Ser. B, 46, 193-227.

Diggle, P. J., Gates, D. J. and Stibbard, A. (1987). A nonparametric estimator for pairwiseinteraction point processes, Biometrika, 74, 763-770.

Diggle, P. J., Fiksel, T., Grabarnik, P., Ogata, Y., Stoyan, D. and Tanemura, M. (1994). On parameter estimation for pairwise interaction processes, Internat. Statist. Rev., 62, 99-117.

Fiksel, T. (1984). Estimation of parametrized pair potentials of marked and nonmarked Gibbsian point processes, Elektronische Informationsverarbeitung und Kybernetika, 20, 270-278.

Fiksel, T. (1988). Estimation of interaction potentials of Gibbsian point processes, Statistics, $19,77-86$.

Gates, D. J. and Westcott, M. (1986). Clustering estimates for spatial point distributions with unstable potentials, Ann. Inst. Statist. Math., 38, 123-135.

Geyer, C. J. and Møller, J. (1993). Simulation procedures and likelihood inference for spatial point processes, Research Report, 260, Mathematical Institute, University of Aarhus, Denmark.

Geyer, C. J. and Thompson, E. A. (1992). Constrained Monte Carlo maximum likelihood for dependent data (with discussion), J. Roy. Statist. Soc. Ser. B, 54, 657-699.

Glötzl, E. (1980a). Bemerkungen zu einer Arbeit von O. K. Kozlov, Math. Nachr., 94, 277-289.

Glötzl, E. (1980b). Lokale Energien und Potentiale für Punktprozesse, Math. Nachr., 96, 195206.

Hall, P. (1988). An Introduction to the Theory of Coverage Processes, Wiley, New York.

Hamilton, W. D. (1971). Geometry for the selfish herd, J. Theoret. Biol., 31, 295-311. 
Hammersley, J. M., Lewis, J. W. E. and Rowlinson, J. S. (1975). Relationships between the multinomial and Poisson models of stochastic processes, and between the canonical and grand canonical ensembles in statistical mechanics, with illustrations and Monte Carlo methods for the penetrable sphere model of liquid-vapour equilibrium, Sankhyā Ser. A, 37, 457-491.

Illingworth, J. and Kittler, J. (1988). A survey of the Hough transform, Computer Vision, Graphics and Image Processing, 44, 87-116.

Jensen, J. L. (1993). Asymptotic normality of estimates in spatial point processes, Scand. J. Statist., 20, 97-109.

Jensen. J. L. and Møller, J. (1991). Pseudolikelihood for exponential family models of spatial point processes, Annals of Applied Probability, 1, 445-461.

Kallenberg, O. (1984). An informal guide to the theory of conditioning in point processes, Internat. Statist. Rev., 52, 151-164.

Kelly, F. P. and Ripley, B. D. (1976). On Strauss's model for clustering, Biometrika, 63, 357-360.

Kendall, W. S. (1990). A spatial Markov property for nearest-neighbour Markov point processes, J. Appl. Probab., 28, 767-778.

Kozlov, O. K. (1976). Gibbsian description of point random fields, Theory Probab. Appl., 21, 339-355.

Lawson, A. (1993). Gibbs sampling putative pollution sources, Tech. Report, Dundee Institute of Technology (in preparation).

Van Lieshout, M. N. M. and Baddeley, A. J. (1995). A nonparametric measure of spatial interaction in point patterns, Statist. Neerlandica (in press).

Mase, S. (1990). Mean characteristics of Gibbsian point processes, Ann. Inst. Statist. Math., 42, 203-220.

Matheron, G. (1975). Random Sets and Integral Geometry, Wiley, New York.

Matthes, K., Warmuth, J. and Mecke, J. (1979). Bemerkungen zu einer Arbeit von X. X. Nguyen und H. Zessin, Math. Nachr., 88, 117-127.

Møller, J. (1989). On the rate of convergence of spatial birth-and-death processes, Ann. Inst. Statist. Math., 41, 565-581.

Møller, J. (1992). Discussion contribution, J. Roy. Statist. Soc. Ser. B, 54, 692-693.

Møller, J. (1994). Markov chain Monte Carlo and spatial point processes, Research Report, 293, Mathematical Institute, University of Aarhus, Denmark.

Moyeed, R. A. and Baddeley, A. J. (1991). Stochastic approximation for the MLE of a spatial point process, Scand. J. Statist., 18, 39-50.

Nguyen, X. X. and Zessin, H. (1979). Integral and differential characterization of the Gibbs process, Math. Nachr., 88, 105-115.

Ogata, Y. and Tanemura, M. (1981). Estimation for interaction potentials of spatial point patterns through the maximum likelihood procedure, Ann. Inst. Statist. Math., 33, 315338.

Ogata, Y. and Tanemura, M. (1984). Likelihood analysis of spatial point patterns, J. Roy. Statist. Soc. Ser. B, 46, 496-518.

Ogata, Y. and Tanemura, M. (1989). Likelihood estimation of soft-core interaction potentials for Gibbsian point patterns, Ann. Inst. Statist. Math., 41, 583-600.

Penttinen, A. (1984). Modelling Interaction in Spatial Point Patterns: Parameter Estimation by the Maximum Likelihood Method, Number 7 in Jyväskylä Studies in Computer Science, Economics and Statistics, University of Jyvaskyla.

Preston, C. J. (1976). Random Fields, Springer, Berlin.

Preston, C. J. (1977). Spatial birth-and-death processes, Bull. Internat. Statist. Inst., 46, 371391.

Ripley, B. D. (1977). Modelling spatial patterns (with discussion), J. Roy. Statist. Soc. Ser. B, 39, 172-212.

Ripley, B. D. (1981). Spatial Statistics, Wiley, New York. Ripley, B. D. (1988). Statistical Inference for Spatial Processes, Cambridge University Press,
U.K.

Ripley, B. D. (1989). Gibbsian interaction models, Spatial Statistics: Past, Present and Future (ed. D. A. Griffiths), 1-19, Image, New York. 
Ripley, B. D. and Kelly, F. P. (1977). Markov point processes, J. London Math. Soc., 15, 188-192.

Rosenfeld, A. and Pfalz, J. L. (1968). Distance functions on digital pictures, Pattern Recognition, 1, 33-61.

Rowlinson, J. S. (1980). Penetrable sphere models of liquid-vapor equilibrium, Adv. Chem. Phys., 41, 1-57.

Rowlinson, J. S. (1990). Probability densities for some one-dimensional problems in statistical mechanics, Disorder in Physical Systems (eds. G. R. Grimmett and D. J. A. Welsh), 261-276, Clarendon Press, Oxford.

Ruelle, D. (1969). Statistical Mechanics, Wiley, New York.

Särkkä, A. (1989). On parameter estimation of Gibbs point processes through the pseudolikelihood estimation method, Research Report, 4, Department of Statistics, University of Jyväskylä, Finland.

Särkkä, A. (1990). Applications of Gibbs point processes: pseudo-likelihood method with comparisons, Research Report, 10, Department of Statistics, University of Jyväskylä, Finland.

Stoyan, D. and Stoyan, H. (1992). Fraktale-Formen-Punktfelder, Akademie Verlag, Berlin.

Stoyan, D., Kendall, W. S. and Mecke, J. (1987). Stochastic Geometry and Its Applications, Wiley, Chichester.

Strauss, D. J. (1975). A model for clustering, Biometrika, 63, 467-475.

Takacs, R. (1983). Estimator for the pair-potential of a Gibbsian point process, Institutsbericht, 238, Institut für Mathematik, Johannes Kepler Universität Linz, Austria.

Takacs, R. (1986). Estimator for the pair potential of a Gibbsian point process, Statistics, 17, $429-433$

Widom, B. and Rowlinson, J. S. (1970). New model for the study of liquid-vapor phase transitions, J. Chem. Phys., 52, 1670-1684. 